\title{
Impact of synthetic and biological immunomodulatory therapy on the duration of 17DD yellow fever vaccine- induced immunity in rheumatoid arthritis
}

Clarissa de Castro Ferreira ${ }^{1,5^{*}}$ (B), Ana Carolina Campi-Azevedo ${ }^{2,5}$, Vanessa Peruhype-Magalhāes ${ }^{2,5}$, Jordana Grazziela Coelho-dos-Reis ${ }^{2,5}$, Lis Ribeiro do Valle Antonelli ${ }^{2,5}$, Karen Torres ${ }^{2,5}$, Larissa Chaves Freire ${ }^{2,5}$, Ismael Artur da Costa-Rocha ${ }^{2,5}$, Ana Cristina Vanderley Oliveira ${ }^{1}$, Maria de Lourdes de Sousa Maia ${ }^{3}$, Sheila Maria Barbosa de Lima ${ }^{3}$, Carla Magda Domingues ${ }^{4}$, Andréa Teixeira-Carvalho ${ }^{2,5}$, Olindo Assis Martins-Filho ${ }^{2,5}$, Lícia Maria Henrique da Mota ${ }^{1}$ and on behalf of the Collaborative Group for Studies of Yellow Fever Vaccine

\begin{abstract}
Background: The 17DD-yellow fever (YF) vaccine induces a long-lasting protective immunity, resulting from humoral and cellular immunological memory. The treatment of rheumatoid arthritis (RA) patients with diseasemodifying anti-rheumatic drugs (DMARD) may affect pre-existing 17DD-vaccine protective immunity and increase the risk of acquiring YF infection. Our goal was to determine whether DMARD would affect the duration of YFspecific protective immunity in RA patients.

Methods: A total of 122 RA patients, previously immunized with the 17DD-YF vaccine $(1-5,5-9$, and $\geq 10$ years) and currently under DMARD therapy, were enrolled in the present investigation. Immunomodulatory therapy encompasses the use of conventional synthetic DMARD alone (cSDMARD) or combines with biological DMARD (cs + bDMARD). A total of 226 healthy subjects were recruited as a control group (CONT). Neutralizing antibody responses were measured by a plaque-reduction neutralization test (PRNT), and cellular immunity was evaluated by an in vitro 17DD-YF-specific peripheral blood lymphoproliferative assay.

Results: The data demonstrated that CSDMARD therapy did not affect the duration of protective immunity induced by the 17DD-YF vaccine compared to that of CONT, as both presented a significant time-dependent decline at 10 years after vaccination. Conversely, cs+bDMARD therapy induced a premature depletion in the main determinants of the vaccine protective response, with diminished PRNT seropositivity levels between 5 and 9 years and impaired effector memory in $\mathrm{CD}^{+} \mathrm{T}$ cells as early as $1-5$ years after 17DD-YF vaccination.

Conclusions: These findings could support changing the vaccination schedule of this population, with the possibility of a planned booster dose upon the suspension of bDMARD in cases where this is allowed, even before 10 years following 17DD-YF vaccination. The benefit of a planned booster dose should be evaluated in further studies.

(Continued on next page)
\end{abstract}

\footnotetext{
* Correspondence: ferreira.clarissa@gmail.com

'Departamento de Reumatologia, Hospital Universitário de Braśilia,

Universidade de Brasília, Brasília, DF, Brazil

${ }^{5}$ Grupo Integrado de Pesquisas em Biomarcadores, Instituto René Rachou,

Fundação Oswaldo Cruz, FIOCRUZ-Minas, Avenida Augusto de Lima, 1715

Barro Preto, Belo Horizonte 30190-002, Brazil

Full list of author information is available at the end of the article
}

(c) The Author(s). 2019 Open Access This article is distributed under the terms of the Creative Commons Attribution 4.0 International License (http://creativecommons.org/licenses/by/4.0/), which permits unrestricted use, distribution, and

reproduction in any medium, provided you give appropriate credit to the original author(s) and the source, provide a link to the Creative Commons license, and indicate if changes were made. The Creative Commons Public Domain Dedication waiver (http://creativecommons.org/publicdomain/zero/1.0/) applies to the data made available in this article, unless otherwise stated. 
(Continued from previous page)

Trial registration: RBR-946bv5. Date of registration: March 05, 2018. Retrospectively registered

Keywords: Immunomodulatory therapy, Yellow fever vaccine, Neutralizing antibodies, Cellular immunity, Rheumatoid arthritis

\section{Background}

Rheumatoid arthritis (RA) is one of the most prevalent chronic autoimmune diseases, and it mainly affects the peripheral joints and promotes synovitis, which may lead to cartilage damage and bone erosion [1]. The prevalence of RA ranges from 0.40 to $1.60 \%$ and 0.46 to $1.00 \%$ in Latin America [2, 3] and Brazil [4, 5], respectively.

In recent decades, significant advances in RA clinical and therapeutic approaches have been reported worldwide, encompassing the use of conventional synthetic and biological strategies, such as pathway inhibitors/antagonists. While effective for controlling RA activity, these immunomodulatory therapies may affect pre-existing immunity to infectious diseases. In a scenario in which immunizations are effective to elicit protective immunity, it becomes relevant to understand the impact that disease-modifying anti-rheumatic drugs (DMARD) has on correlates of protection acquired upon vaccination $[6,7]$. The ability of DMARD to modify or affect pre-existing vaccine-induced protective immunity, including the function of memory $\mathrm{T}$ and $\mathrm{B}$ cells and, as a consequence, yellow fever (YF)-specific neutralizing antibody levels, has already been reported [8]. There is a paucity of data available regarding the impact of DMARD on the duration of YF vaccine-induced immunological memory developed by RA patients.

The recent YF outbreaks in Angola (2016) and Brazil (2017/2018) [9, 10] brought about a relevant question regarding the impact that DMARD may have on RA patients previously immunized with the 17DD-YF vaccine. Assessing the duration of the immune responses triggered by YF vaccines can provide insights into elucidating the vulnerability to YF infection of RA patients under DMARD therapy.

In this context, the aim of the present study was to verify whether conventional synthetic or biological DMARD impact the cellular and humoral immunological memory of RA patients previously immunized with the 17DD-YF vaccine. These findings may be useful when making clinical decisions regarding YF vaccination in RA patients.

\section{Methods}

\section{Subjects}

Between September 17, 2014, and December 06, 2016, 136 adult patients ( $\geq 18$ years) who met ACR classification criteria for RA were enrolled in this open-label, parallel cohort, single-center study. Patients had received a single dose of the 17DD-YF vaccine and time after vaccination estimated according to their vaccination card records; some patients have been inadvertently vaccinated before starting the DMARD therapy; all patients were residents of the metropolitan area of Brasilia, DF, Brazil, and received medical care at the University Hospital of Brasilia, University of Brasilia. Fifteen patients were excluded due to their 17DD-YF vaccination records showing $<1$ year, $>30$ years $(n=12)$, or missing data $(n$ $=3$ ). The final RA group comprised 121 subjects, 113 females and 9 males, aged 23 to 86 years, categorized into two subgroups based on whether they were under immunotherapy with conventional synthetic disease-modifying anti-rheumatic drugs (csDMARD, $n=$ 73) or under combined immunotherapy with csDMARD plus biological disease-modifying anti-rheumatic drugs (cs+bDMARD, $n=48$ ). The csDMARD and cs + bDMARD subgroups were further segregated according to the time after 17DD-YF vaccination, as follows: $1-5$ years, $>5-9$ years, and $\geq 10$ years. Details regarding the demographic features, clinical records, and immunomodulatory therapy dosages are provided in Table 1.

The control group of healthy subjects included 226 volunteers, 121 males and 59 females; the subjects were aged 18-82 years and categorized into five subgroups referred to as non-vaccinated subjects $\mathrm{NV}($ day 0$)$ and primary vaccinated $\mathrm{PV}($ day30-45) and three groups of healthy controls (CONT); the controls were categorized according to the time after their 17DD-YF vaccination: $\operatorname{CONT}(1-5$ years $), \operatorname{CONT}(>5-9$ years $)$, and $\operatorname{CONT}(\geq 10$ years). Whole blood samples were collected from each volunteer: $5 \mathrm{~mL}$ without anticoagulant for the plaque-reduction neutralization test (PRNT) and $20 \mathrm{~mL}$ in heparin to isolate peripheral blood mononuclear cells (PBMC) for analyses of cellular immunity. A detailed compendium of the study population and methods are provided in Fig. 1.

This study was approved by the Ethics Committee for studies with human subjects at Instituto René Rachou FIOCRUZ (CPqRR \# 180911). All subjects gave written informed consent in accordance with the Declaration of Helsinki.

\section{YF-neutralizing antibody test (PRNT)}

The 17DD-YF-neutralizing antibody test (PRNT) was performed as previously described [11, 12]. The assays 
Table 1 Demographic features, clinical records, and immunomodulatory therapy of the AR population

\begin{tabular}{|c|c|c|c|c|c|c|}
\hline \multirow[t]{2}{*}{ Parameters } & \multicolumn{3}{|l|}{$\begin{array}{l}\text { csDMARD } \\
n=73\end{array}$} & \multicolumn{3}{|l|}{$\begin{array}{l}c s+b D M A R D \\
n=48\end{array}$} \\
\hline & $\begin{array}{l}1-5 \text { years } \\
n=18\end{array}$ & $\begin{array}{l}>5-9 \text { years } \\
n=37\end{array}$ & $\begin{array}{l}\geq 10 \text { years } \\
n=18\end{array}$ & $\begin{array}{l}1-5 \text { years } \\
n=10\end{array}$ & $\begin{array}{l}>5-9 \text { years } \\
n=25\end{array}$ & $\begin{array}{l}\geq 10 \text { years } \\
n=13\end{array}$ \\
\hline Gender (F/M) & $17 / 01$ & $35 / 02$ & $15 / 03$ & 09/01 & $23 / 02$ & $13 / 00$ \\
\hline Age (years) & $58(31-78)$ & $53(26-86)$ & $49(23-81)$ & $55(28-75)$ & $60(40-82)$ & $54(29-80)$ \\
\hline Disease duration (months) & $99(12-432)$ & $114(12-348)$ & $84(12-480)$ & $210(96-298)$ & $144(72-370)$ & 168 (96-288) \\
\hline \multicolumn{7}{|l|}{ csDMARD dose } \\
\hline MTX (2.5- 25 mg/week) & $17 \mathrm{mg}(14 / 18)$ & $16 \mathrm{mg}(35 / 37)$ & $18 \mathrm{mg}(12 / 18)$ & $23 \mathrm{mg}(05 / 10)$ & 17 mg (14/25) & 17 mg (04/13) \\
\hline LEF (standard dose/day) & $20 \mathrm{mg}(06 / 18)$ & $20 \mathrm{mg}(19 / 37)$ & $20 \mathrm{mg}(09 / 18)$ & $20 \mathrm{mg}(05 / 10)$ & $20 \mathrm{mg}(12 / 25)$ & $20 \mathrm{mg}(06 / 13)$ \\
\hline SSZ (1000-3000 mg/day) & $1500 \mathrm{mg}(02 / 18)$ & - & 2300 mg (03/18) & - & $1000 \mathrm{mg}(02 / 25)$ & 1200 mg (05/13) \\
\hline AML (150-400 mg/day) & 275 mg (04/18) & $340 \mathrm{mg}(05 / 37)$ & - & - & 150 mg/day (01/25) & 275 mg/day (02/13) \\
\hline AZA (2-3 mg/kg/day) & - & - & - & $150 \mathrm{mg}(01 / 10)$ & - & - \\
\hline CYC (3-5 mg/kg/day) & - & - & - & - & - & 200 mg (01/13) \\
\hline \multicolumn{7}{|l|}{ bDMARD dose } \\
\hline ADA (standard dose/eow) & - & - & - & 40 mg (01/10) & $40 \mathrm{mg}(02 / 25)$ & 40 mg (01/13) \\
\hline CTZ (standard dose/month) & - & - & - & 400 mg (02/10) & - & $400 \mathrm{mg}(01 / 13)$ \\
\hline ETN (standard dose/week) & - & - & - & $50 \mathrm{mg}(05 / 10)$ & $50 \mathrm{mg}(05 / 25)$ & $50 \mathrm{mg}(04 / 13)$ \\
\hline GOL (standard dose/month) & - & - & - & - & $50 \mathrm{mg}(02 / 25)$ & $50 \mathrm{mg}(03 / 13)$ \\
\hline IFX (3-5 mg/kg/e8w) & - & - & - & 300 mg (01/10) & 233 mg (06/25) & - \\
\hline TCZ (8 mg/kg/month) & - & - & - & - & $496 \mathrm{mg}(05 / 25)$ & $480 \mathrm{mg}(02 / 13)$ \\
\hline ABT (10 mg/kg/month) & - & - & - & - & 750 mg (03/25) & 750 mg (01/13) \\
\hline RTX (standard dose/e6m) & - & - & - & $1000 \mathrm{mg}(01 / 10)$ & $1000 \mathrm{mg}(02 / 25)$ & 1000 mg (01/13) \\
\hline \multicolumn{7}{|l|}{ GC } \\
\hline PDN (2.5-40 mg/day) & $7.5 \mathrm{mg}(04 / 18)$ & $13.6 \mathrm{mg}(06 / 37)$ & $8.2 \mathrm{mg}(07 / 18)$ & 6.7 mg (03/10) & $10.8 \mathrm{mg}(06 / 25)$ & $7.0 \mathrm{mg}(05 / 13)$ \\
\hline
\end{tabular}

Age is expressed as median (min-max). Disease duration in months is expressed as median (min-max). Immunomodulatory therapeutic dosages are provided for each drug and as mean dose/group

cSDMARD conventional synthetic disease-modifying anti-rheumatic drugs, cs+bDMARD combined conventional synthetic and biological disease-modifying antirheumatic drugs, CG glucocorticoid, $F$ female, $M$ male, MTX methotrexate, LEF leflunomide, SSZ sulfasalazine, $A M L$ anti-malarial drugs (hydroxychloroquine and chloroquine phosphate), AZA azathioprine, CYC ciclosporin, ADA adalimumab, CTZ certolizumab, ETN etanercept, GOL golimumab, IFX infliximab, TCZ tocilizumab, $A B T$ abatacept, $R T X$ rituximab, PDN prednisone, eow every other week, e8w every 8 weeks, e6m every 6 months

were carried out at Laboratório de Tecnologia Virológica, Bio-Manguinhos (LATEV, FIOCRUZ-RJ, Brazil), and the results are expressed as a reverse of the samples' dilution. The samples were considered seropositive when the PRNT levels were higher than the serum dilution 1:50.

\section{Analysis of cellular immunity}

PBMC $\left(1.0 \times 10^{6} /\right.$ well $)$ were incubated for $144 \mathrm{~h}$ at $37^{\circ} \mathrm{C}$ in a $5 \% \mathrm{CO}_{2}$ humidified atmosphere, in the absence (Control/ $\mathrm{CC}$ ) or presence of 17DD-YF antigen (17DD-YF Ag), as described previously [13]. Following the long-term incubation, the PBMC were stained with live/dead dye and a cocktail of monoclonal antibodies (mAbs), including anti-CD4/ (RPA-T4)/FITC, anti-CD8/(SK1)/PerCP-Cy5.5, anti-CD27/ (M-T271)/PE, anti-CD45RO/(UCHL1)/PE-Cy, anti-CD3/ (SK7)/APC-Cy7, anti-IgD/(IA6-2)/FITC, anti-CD27/ (M-T271)/PE, and anti-CD19/(HIB19)/PerCP for the analysis of the $\mathrm{T}$ and $\mathrm{B}$ cell phenotypic memory status.
In parallel, PBMC were stained for the functional analysis of $\mathrm{T}$ and $\mathrm{B}$ cells. Cells were first incubated with anti-CD3/(UCHT1)/Qdot605, anti-CD4/(GK1.5)/APCe-Fluor780, anti-CD8/(SK1)/PerCP, and anti-CD19/ (HIB19)/Alexa-Fluor700. Then, surface-stained PBMC were subjected to a fix/perm procedure and stained with anti-IFN- $\gamma /$ (clone B27)/Alexa-Fluor488, anti-IL-5/ (JES1-39D10)/PE, anti-IL-10/(JES3-19F1)/APC, and anti-TNF- $\alpha /($ clone MAb11)/PE-Cy7. After staining, cells were fixed, and acquisition was carried out on an LSR Fortessa Flow Cytometer.

A total of 100,000 events were acquired per sample, and gating strategies were employed for phenotypic and functional memory using the FlowJo software, version 9.3.2, as previously described [13]. Four memory $\mathrm{T}$ cell subsets [naive/(NCD4 and $\mathrm{NCD} 8) / \mathrm{CD} 27^{+} \mathrm{CD} 45 \mathrm{RO}^{-}$, early effector memory/(eEfCD4 and eEfCD8)/ $\mathrm{CD}_{27}{ }^{-} \mathrm{CD}_{45 \mathrm{RO}^{-}}$, central memory/(CMCD4 and $\mathrm{CMCD} 8) / \mathrm{CD} 27^{+} \mathrm{CD} 45 \mathrm{RO}^{+}$, and effector memory/ 


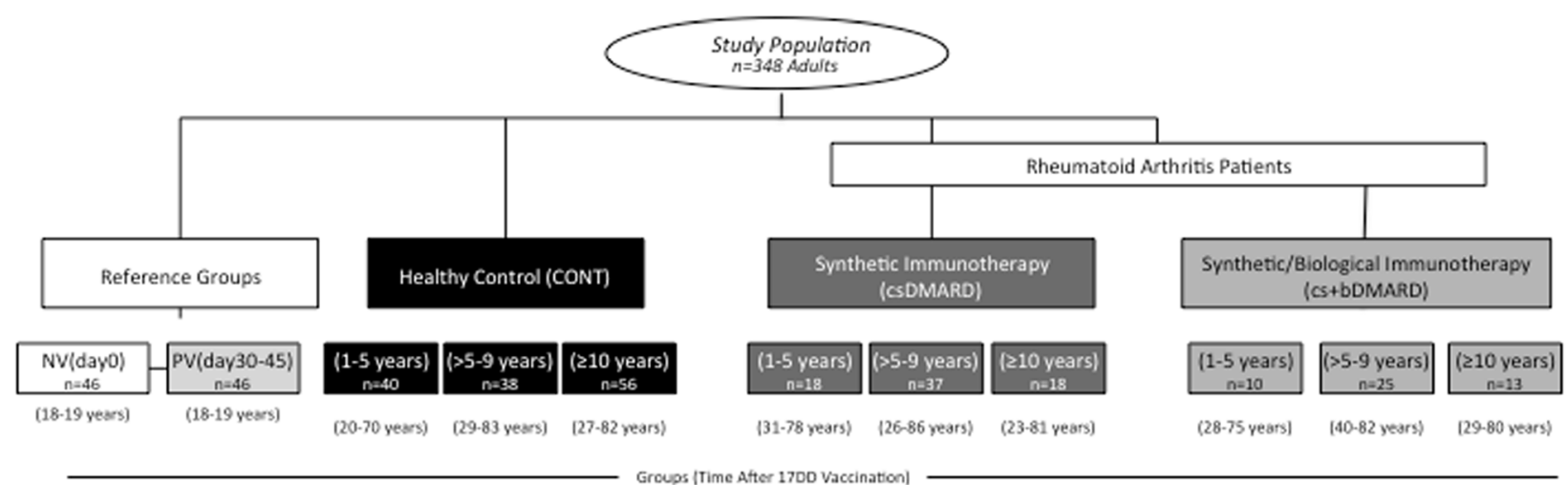

Grougs (Time After 1700 Vaccination)

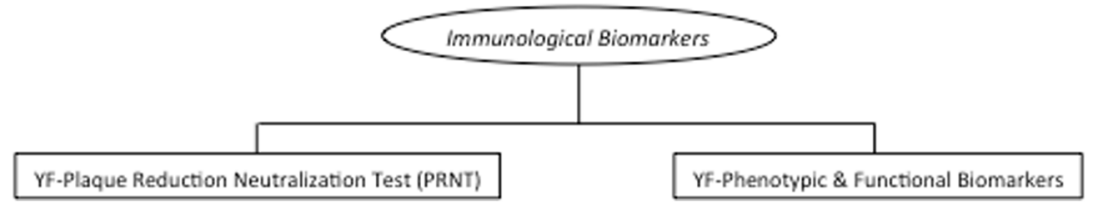

Fig. 1 Compendium of the study population. A total of 348 adults were enrolled in the present investigation. One hundred and twenty-one adult RA patients with previous records of 17DD-YF vaccination were enrolled. Patients were first categorized into two subgroups, referred to as synthetic immunotherapy (csDMARD) or combined immunotherapy (cs+bDMARD) based on whether they were under current treatment with DMARDs or DMARDs combined with TNF-a inhibitors (adalimumab/ADA, certolizumab/CTZ, etanercept/ETN, golimumab/GOL, or infliximab/IFX), IL-6 antagonists (tocilizumab/TCZ), T lymphocyte co-stimulation modulators (abatacept/ABT), or anti-B-cell mAbs (rituximab/RTX); the patients were further categorized according to the time after 17DD vaccination as follows: CSDMARD (1-5 years), csDMARD (> 5-9 years), csDMARD ( $\geq 10$ years), and cs+bDMARD (1-5 years), cs+bDMARD ( $>5-9$ years), cs+bDMARD ( $\geq 10$ years). The control group of the healthy subjects included 226 participants categorized into five subgroups referred as non-vaccinated subjects NV(day0), PV(day30-45), and three groups of volunteers, categorized according to the time after 17DD-YF vaccination and referred to as CONT(1-5 years), CONT(> 5-9 years), and CONT( $\geq 10$ years). Detailed descriptions of the study groups are provided in the "Methods" section. Immunological biomarker analyses, including YF plaquereduction neutralization test (PRNT) and YF phenotypic and functional biomarkers, were performed for each participant

(EMCD4 and EMCD8)/CD27 $\left.{ }^{-} \mathrm{CD}^{2} 5 \mathrm{RO}^{+}\right]$and three memory B cell subsets [naïve/(NCD19)/CD27 ${ }^{-} \operatorname{IgD}^{+}$, non-classical memory $/(\mathrm{nCMCD} 19) / \mathrm{CD}^{-} 7^{+} \mathrm{IgD}^{+}$, and classical memory/(CMCD19)/CD27 ${ }^{+} \mathrm{IgD}^{-}$] were quantified. Cytokine ${ }^{+}$cells were also quantified (TNF- $\alpha$, IFN- $\gamma$, IL-10, and IL-5 for T cells and TNF- $\alpha$, IL-10, and IL-5 for $\mathrm{B}$ cells). The results were reported as the 17DD-YF $\mathrm{Ag} / \mathrm{CC}$ index, computed as the frequency of cells observed in the 17DD-YF stimulated culture (17DD-YF Ag) divided by the respective control culture (CC). The characterization of phenotypic and functional features of PBMC has been performed after 17DD-YF-specific in vitro stimuli, and the results are expressed as stimulation index, taking the results from the control culture intrinsic for the same individual as a baseline.

\section{Multiparameter data mining strategies}

Data analyses were carried out employing a set of strategies including conventional statistical, biomarker signature analysis, Venn diagram assembling, and overlaid signature curves.
Conventional statistical approaches were used for comparative analysis with the reference groups $\mathrm{NV}$ (day0) and PV(day30-45). For this purpose, the mean value of each study group (CONT, csDMARD, and cs+bDMARD) was compared with the $95 \%$ CI of the reference groups [NV(day0) and PV(day30-45)]. The differences demonstrated by the mean values outside the $95 \%$ CI were considered significant $(p<$ 0.05 ) and highlighted by letters "a" and "b" compared to NV(day0) or PV(day30-45). Biomarker signature analysis was carried out as described previously [14], using the global median value of $17 \mathrm{DD}-\mathrm{YF} \mathrm{Ag} / \mathrm{CC}$ index for each biomarker as the cut-off to define "low" or "high" 17DD-YF Ag/CC index. The biomarker signatures of $\mathrm{NV}($ day 0$)$ and $\mathrm{PV}($ day30-45) were overlapped as the reference curves, and those biomarkers for which more than $50 \%$ of samples were above the cut-off index were selected for further identification of biomarkers upregulated selectively by the 17DD-YF vaccine using the Venn diagram analysis (http://bioin formatics.psb.ugent.be/webtools/Venn/). The selected 
set of biomarkers identified early after 17DD-YF vaccination in the PV(day30-45) group was underscored in a bold font format. These attributes were employed for comparative analyses among those biomarkers by overlaid signature curves in which the 50th percentile defined significant differences for each study group (CONT, csDMARD, and cs+bDMARD) at distinct time points after 17DD-YF vaccination.

\section{Results}

Early decrease of 17DD-YF-neutralizing antibodies is observed in RA patients undergoing combined synthetic/ biological immunomodulatory therapy

The analysis of PRNT levels is presented in Fig. 2. Data are reported as ranges of PRNT levels and proportion of PRNT seropositivity (serum dilution > 1:50). The results demonstrated a decrease of PRNT levels over time after 17DD-YF vaccination in all study groups (CONT, csDMARD, and cs+bDMARD) compared to the reference group PV(day30-45) (Fig. 2a). The PRNT seropositivity rate reaches critical values in CONT (71\%) and csDMARD (72\%) at 10 years after vaccination (Fig. 2b). Conversely, data demonstrated that combined immunotherapy has a deleterious impact on the PRNT seropositivity rate. In fact, in the cs+bDMARD group, the decrease in the PRNT seropositivity rate occurs earlier compared to CONT and SDMARD, reaching critical values $(76 \%)$ at $>5-9$ years after $17 \mathrm{DD}-\mathrm{YF}$ vaccination (Fig. 2b).

\section{Distinct duration of 17DD-YF-specific phenotypic memory biomarkers is observed in RA patients upon immunomodulatory therapy}

The profile of 17DD-specific phenotypic memory biomarkers is shown in Fig. 3. Our data show that patients with RA undergoing immunomodulatory therapy (csDMARD or cs+bDMARD) presented a distinct overall profile of phenotypic memory biomarkers, characterized by increased levels of eEfCD4 and decreased levels of CMCD4, NCD19, and nCMCD19 compared to CONT. The cs+bDMARD group presented a particular decrease of EMCD4, CMCD8, and EMCD8 as early as 1-5 years after 17DD-YF vaccination compared to CONT and csDMARD (Fig. 3).

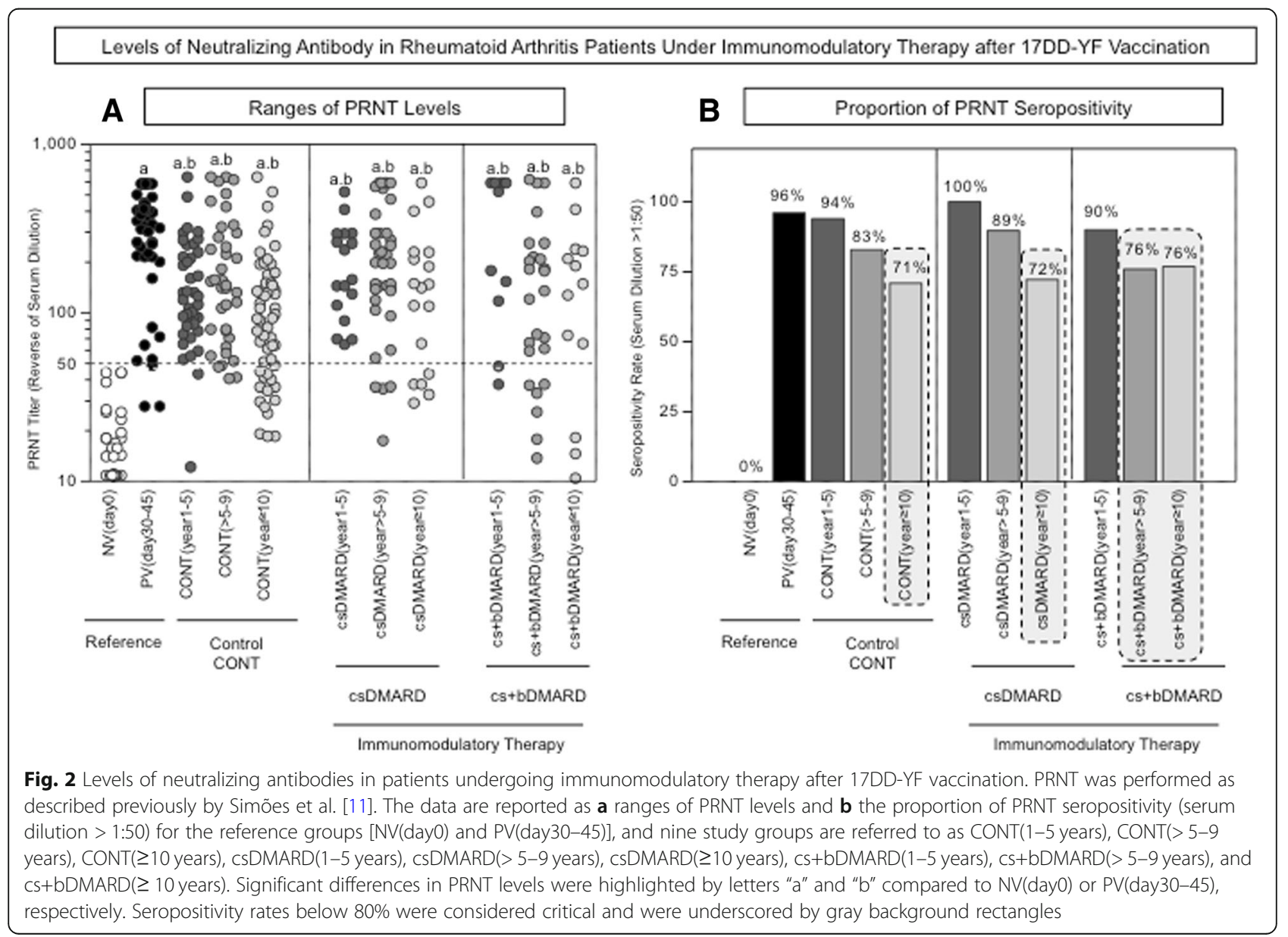




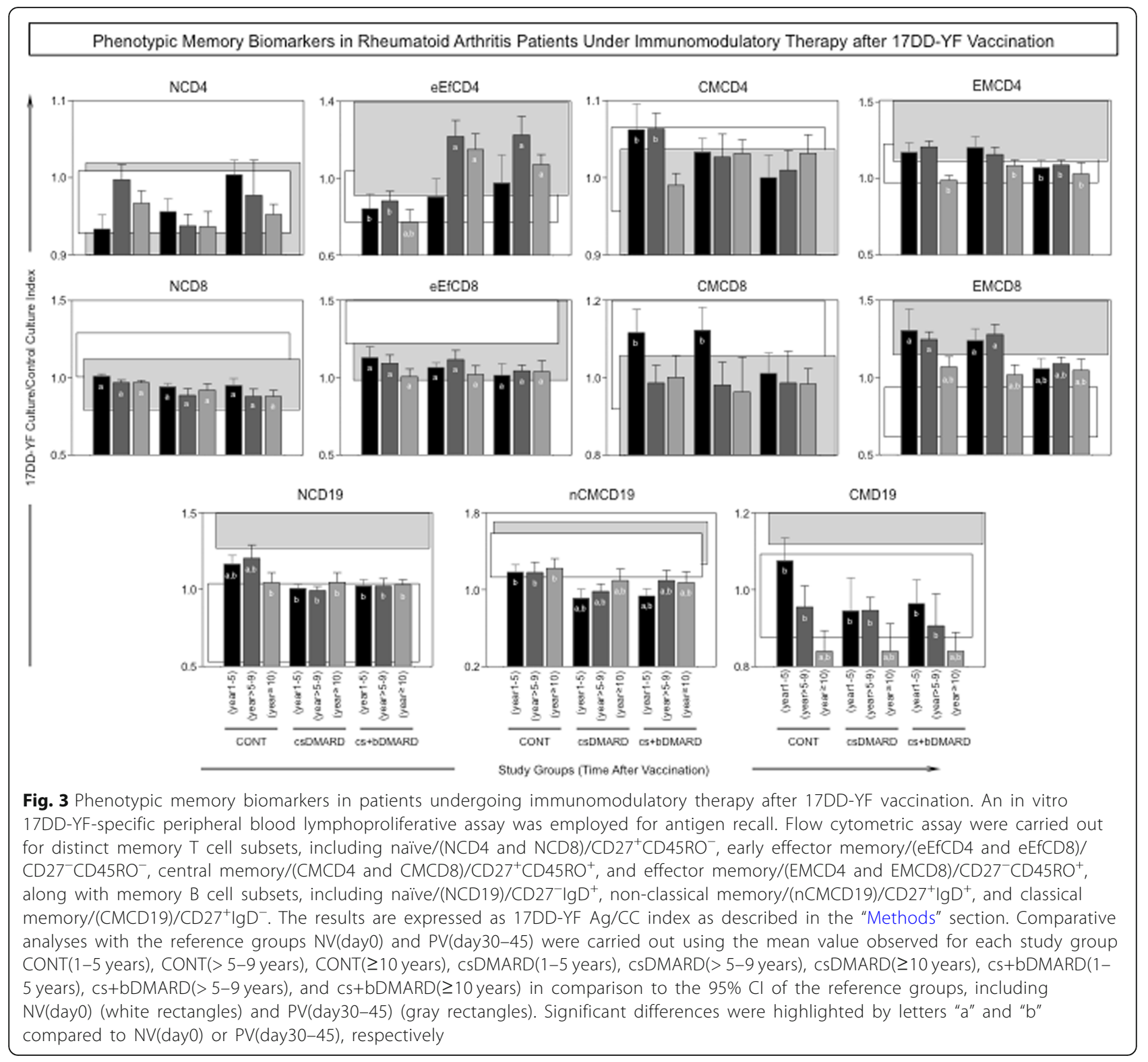

Early decrease of 17DD-YF-specific functional memory biomarkers is observed in RA patients receiving synthetic/biological combined immunomodulatory therapy

The profile of 17DD-specific phenotypic biomarkers is shown in Fig. 4. Patients with RA undergoing immunomodulatory therapy (csDMARD or cs+bDMARD) presented a distinct pattern of functional biomarkers, particularly exemplified by decreased levels of IL-5CD8 and IL-10CD19 compared to CONT. Noteworthy was the decrease of IFNCD4, IFNCD8, TNFCD4, and TNFCD19, along with lower levels of IL-5CD4 observed in the cs+bDMARD group as early as 1-5 years after $17 \mathrm{DD}-\mathrm{YF}$ vaccination compared to CONT and csDMARD (Fig. 4).
17DD-YF-specific memory biomarker signatures in RA patients undergoing immunomodulatory therapy

The biomarker signature has been proposed previously [14] as a reliable approach to characterize the overall profile of immune responses in patients vaccinated with 17DD vaccines. This approach allows the identification of the most relevant biomarkers among a range of attributes. For this purpose, we performed a comparative analysis between the biomarker signatures of the reference groups $\mathrm{NV}($ day0) and $\mathrm{PV}($ day30-45) to identify biomarkers selectively elicited early after vaccination (Fig. 5a). Using this multiparameter approach and Venn diagrams (Fig. 5b), we identified a set of 17DD-YF-specific biomarkers useful for further monitoring the memory signature of the study groups (CONT, 


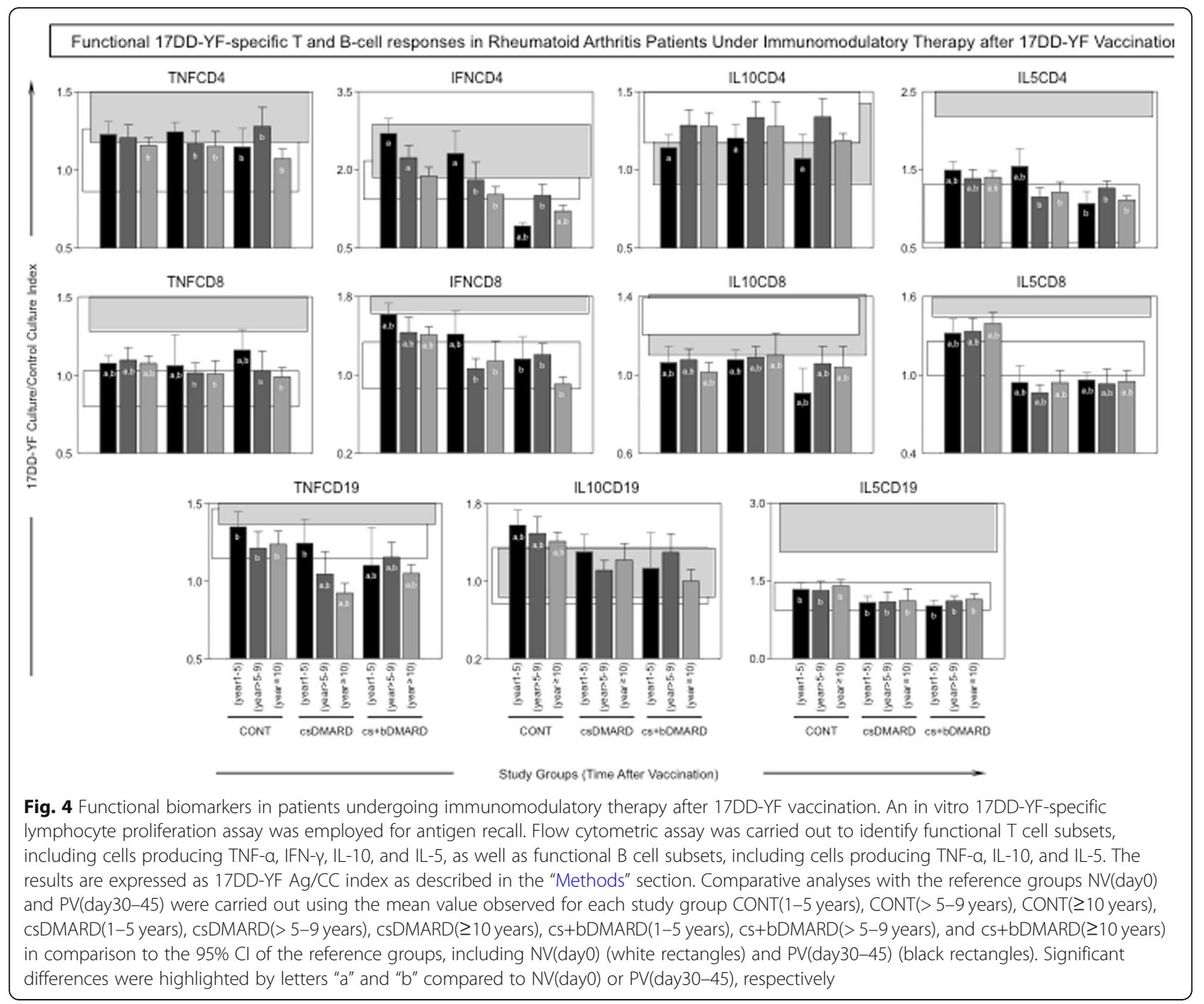

csDMARD, and cs+bDMARD) over time after vaccination. A set of nine phenotypic and functional biomarkers (EMCD4, EMCD8, CMCD19, IFNCD4, TNFCD4, IL-5CD4, IFNCD8, TNFCD8, and IL-5CD8) was identified as selectively predominant in the biomarker signatures of the PV(day30-45) (Fig. 5b).

This set of biomarkers was then employed to follow up with the phenotypic/functional memory signatures among the biomarkers frequently observed above the 50th percentile over time after 17DD-YF vaccination for each study group (CONT, csDMARD, and cs +bDMARD) (Fig. 6). Among these biomarkers, special attention was given to EMCD8 and IL-5CD4, previously reported as the top two biomarkers to monitor immunological memory to the 17DD-YF vaccine [15]. Overlaid biomarker signatures were plotted for comparative analysis among groups at distinct time points after vaccination, including $1-5$ years - Fig. $6 \mathrm{a},>5-9$ years-Fig. $6 \mathrm{~b}$, and $\geq 10$ years-Fig. $6 c$. The results demonstrated that
CONT and csDMARD presented a progressive decrease in the number of biomarkers above the 50th percentile, reaching a critical profile with an absence of EMCD8 at 10 years after vaccination. Conversely, the cs+bDMARD group displayed an overall shortage on the number of biomarkers above the 50th percentile, with an absence of EMCD8 as early as $1-5$ years after $17 D D-Y F$ vaccination.

Venn diagrams were constructed and corroborated the finding that RA patients undergoing combined immunomodulatory therapy did not share either of the top two biomarkers (EMCD8 and IL-5CD4), considered correlates of protection elicited by the 17DD vaccination, with the PV(day30-45) reference group. The CONT and csDMARD groups presented sustained relevant levels of EMCD8 up to $>5-9$ years but showed a critical decrease in this cell subset at 10 years after 17DD-YF vaccination. On the other hand, the cs+bDMARD group displayed a premature loss of the top two biomarkers as early as 15 years after vaccination. 


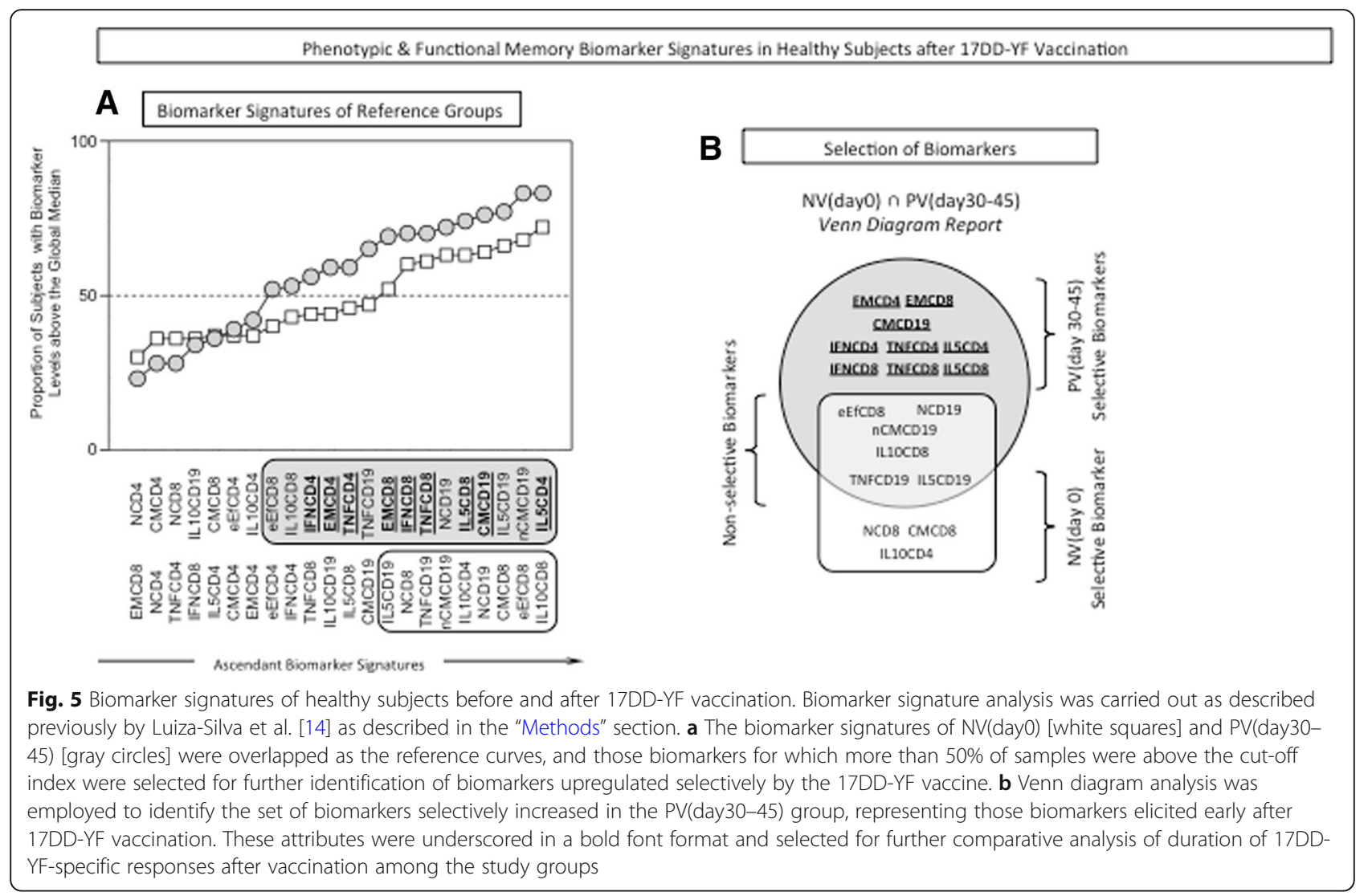

\section{Discussion}

This is an innovative investigation that has been performed to evaluate the impact of immunomodulatory therapy on the duration of the YF vaccine response in RA patients who were previously given the 17DD vaccine.

In general, both immunomodulatory therapies (csDMARD or cs+bDMARD) induced an increase in eEfCD4; a decrease in CMCD4, NCD19, and nCMCD19; and diminished IL-5CD8 and IL-10CD19 compared to CONT. These cell subsets have not been nominated as the most relevant correlates of protection in adults that received the 17DD-YF vaccine [15]. Overall, less significant changes in cellular immunity were observed in RA patients undergoing therapy with csDMARD, who presented an overall similar profile to CONT. Conversely, the evidence demonstrated that therapy with cs +bDMARD has a strong impact on vaccine-induced 17DD-YF-specific memory $\mathrm{T}$ and $\mathrm{B}$ cell responses. Notably, RA patients receiving cs+bDMARD presented a decrease in PRNT levels at $>5-9$ years post-vaccination and a decrease in cellular memory-related markers (EMCD4, CMCD8, EMCD8, IFNCD4, IFNCD8, TNFCD4 and TNFCD19, and IL-5CD4) as early as 1-5 years after vaccination compared to the CONT and csDMARD groups. In association with the PRNT,
EMCD8 and IL-5CD4 were recently nominated as the most relevant biomarkers to follow up with the immune response over time after the 17DD-YF vaccination [15]. The present results corroborate these two correlates of memory response upon 17DD-YF vaccination.

Neutralizing antibodies have long been known to provide protection against challenge with a wild-type virus [16]. The protective role of neutralizing antibodies induced by the YF vaccine has been estimated from dose-response studies carried out in experimental models that were challenged with a virulent YF virus after immunization $[17,18]$. In this sense, the PRNT is considered the most sensitive and specific assay for the quantification of neutralizing antibodies, as well as the reference method for assessing the protective immune response after vaccination [11]. Neutralizing antibodies are induced within 30 days in approximately $98 \%$ healthy adults that received the 17D and 17DD-YF vaccines [19]. Although the neutralizing antibodies are long-lasting, a progressive decrease in the PRNT levels is observed over time after YF vaccination, with approximately $25-30 \%$ of vaccine recipients presenting seroreversion after 10 years of vaccination, suggesting the need of a booster dose to maintain the protective immunity $[13,20,21]$. Our findings corroborate these studies, as demonstrated by the evidence that both CONT and csDMARD groups 


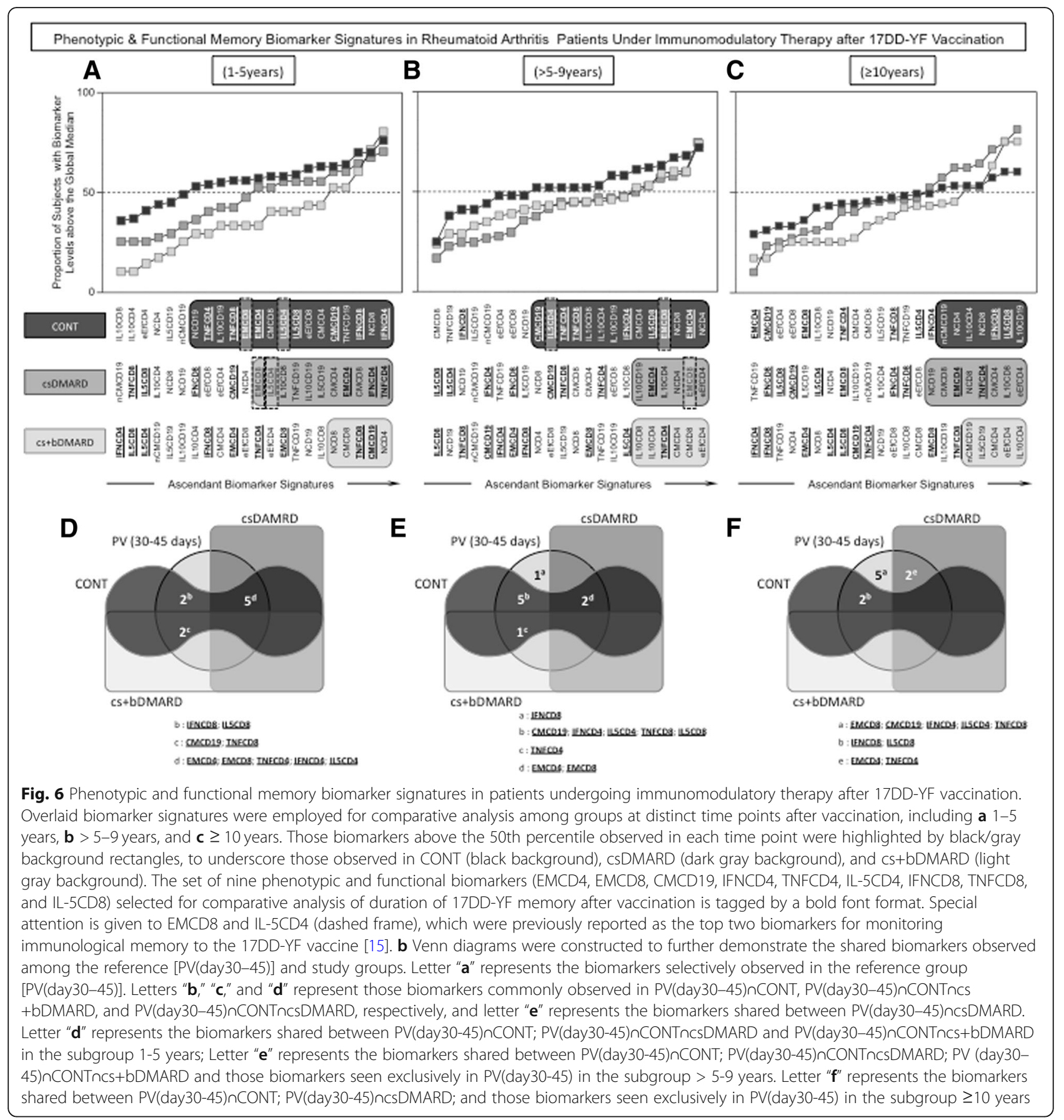

presented a decrease in PRNT levels and in seropositivity rates over time, reaching critical values after 10 years post-vaccination. The earlier loss of humoral response triggered by cs+bDMARD was confirmed by the critical decrease in PRNT seropositivity rate to $76 \%$, observed at >5-9 years post-vaccination in the RA patients undergoing conventional synthetic plus biological immunotherapy schemes; this outcome contrasts with the standard decline observed in CONT and csDMARDs after 10 years of $17 \mathrm{DD}-\mathrm{YF}$ vaccination. In the light of this information, the possibility of a planned booster dose upon suspension of bDMARD should be considered as a strategy to overcome the impaired levels of YF-specific memory-related responses in specific RA cases.

Several studies have reported in detail the development of the cellular immune response after YF vaccination and characterized the phenotypic and functional changes that contribute to the establishment of effector memory $[13,14,22-25]$. Both $\mathrm{CD}^{+}$and $\mathrm{CD}^{+} \mathrm{T}$ cells 
respond strongly to the YF vaccine [16]. It is expected that $\mathrm{CD}^{+} \mathrm{T}$ cells would act primarily to support the production of neutralizing antibody responses. $\mathrm{CD} 8^{+} \mathrm{T}$ cells also respond upon YF vaccination and are considered necessary to recognize and eliminate virus-infected cells. Following the initial peak of $\mathrm{CD}^{+} \mathrm{T}$ cells that occurs early after $\mathrm{YF}$ vaccination, the $\mathrm{CD}^{+} \mathrm{T}$ cells begin to differentiate into long-lived memory with a polyfunctional phenotype. Effector memory $\mathrm{CD}^{+} \mathrm{T}$ cells have been considered one of the top two biomarkers for monitoring the long-lasting cellular immunity triggered by the 17DD-YF vaccine [15]. In the present study, the cs +bDMARD group displayed an early loss of cellular memory to 17DD-YF vaccine, as demonstrated by reduced levels of CMCD4, CMCD8, EMCD4, and EMCD8.

When analyzing the cytokine profile, it is observed that the cs+bDMARD group presents an early and marked decrease in IFNCD4, IFNCD8, TNFCD4, TNFCD19, and IL5CD4, important markers of the immune response triggered by the YF-17DD vaccine. Increased levels of IFN- $\gamma$, TNF- $\alpha$, and IL- 5 have been reported previously [14, 26, 27]. Particularly, TNF has been suggested as a biomarker with a pivotal role in the 17DD-YF vaccine-induced immunity. The prominent participation of TNF produced by neutrophils, monocytes, and $\mathrm{CD} 4^{+} \mathrm{T}$ cells is necessary for the establishment of protective immunity following YF-17DD primary vaccination, free of adverse events [27]. In this sense, it has been demonstrated that children not responding to $17 \mathrm{DD}-\mathrm{YF}$ primary vaccination presented a deficiency in the synthesis of TNF by neutrophils and monocytes [14]. Moreover, it has been shown that a decreased production of TNF, mainly by monocytes and $\mathrm{CD}^{+}{ }^{+} \mathrm{T}$ cells, is associated with the occurrence of severe adverse events after 17DD-YF primary vaccination [28].

In a systematic review evaluating the efficacy of vaccines in patients using immunosuppressant therapy, it was noted that the use of TNF- $\alpha$ antagonists in combination with methotrexate was associated with a reduction of immunogenicity of influenza and pneumococcal vaccines $[8,29]$. Similarly, the use of RTX also led to a similar deleterious impact on the vaccine response to influenza and pneumococcal vaccines [29-32]. On the other hand, in general, AML, SSZ, and LEF did not alter the immunogenicity of these vaccines [31, 32]. These findings are consistent with those found in this study.

The strengths of the study are the simultaneous analysis of cellular and humoral analysis of RA patients previously immunized with the 17DD-YF vaccine and currently using immunosuppressive drugs. Moreover, the csDMARDs and cs+bDMARDS groups have a considerable sample size, differing from previous investigations. The present study has some limitations, considering that it is a cross-sectional investigation that enrolled a convenient sample of RA patients, several of them with previous therapeutic schemes, making it difficult to analyze the impact of each class of DMARD. Furthermore, as this is a cross-sectional study, it does not allow the analysis of the temporal dynamics of the immune response induced by the 17DD-YF vaccine in each individual.

Together, our findings showed that RA patients undergoing treatment with cs+bDMARD have a shorter duration of 17DD-YF vaccine-induced immunity. Based on these results, we suggest that a planned booster dose should be provided to RA patients, especially those residents or travelers to YF-endemic regions. The Brazilian Societies of Rheumatology, Tropical Medicine and Immunization have issued a technical note recommending YF vaccination for patients with immune-mediated rheumatic diseases who are at risk of YF, including those undergoing low immunosuppression or even those under cs+bDMARD therapy for whom the discontinuation of medication is allowed. [33] This vaccination strategy would boost YF-17DD immune responses and ensure safe vaccination, allowing the subsequent return of immunomodulatory therapy after safe immunization.

\section{Conclusions}

csDMARD therapy did not affect the duration of protective immunity induced by the 17DD-YF vaccine compared to that of CONT, as both presented a significant time-dependent decline at 10 years after vaccination. Conversely, cs+bDMARD therapy induced a premature depletion in the main determinants of the vaccine protective response, with diminished PRNT seropositivity levels between 5 and 9 years and impaired effector memory in CD8+ T cells as early as $1-5$ years after $17 \mathrm{DD}-\mathrm{YF}$ vaccination.

These findings could support changing the vaccination schedule of this population, with the possibility of a planned booster dose upon the suspension of bDMARD in cases where this is allowed, even 10 years following 17DD-YF vaccination. The benefit of a planned booster dose should be evaluated in further studies.

\footnotetext{
Abbreviations

e6m: Every 6 months; e8w: Every 8 weeks; eow: Every other week;

ABT: Abatacept; ACR: American College of Rheumatology; ADA: Adalimumab; AML: Anti-malarial; AZA: Azathioprine; CC: Control culture; CM: Central memory; CONT: Control group; cs+bDMARD: Conventional synthetic plus biological DMARD; cSDMARD: Conventional synthetic DMARD; CTZ: Certolizumab; CYC: Ciclosporin; DMARD: Disease-modifying antirheumatic drugs; eEf: Early effector memory; EM: Effector memory; ETN: Etanercept; GOL: Golimumab; IFX: Infliximab; LEF: Leflunomide; mAbs: Monoclonal antibodies; MTX: Methotrexate; N: Naïve; nCM: Nonclassical memory; NV: Non-vaccinated; PBMC: Peripheral blood mononuclear cells; PDN: Prednisone; PRNT: Plaque-reduction neutralization test; PV: Primary vaccinated; RA: Rheumatoid arthritis; RTX: Rituximab; SSZ: Sulfasalazine; TCZ: Tocilizumab; TNF: Tumor necrosis factor; YF: Yellow fever
} 


\section{Acknowledgements}

The authors thank the Program for Technological Development in Tools for Health-RPT-FIOCRUZ for the use of the flow cytometry facility. The authors thank Fundação de Amparo à Pesquisa do Estado de Minas Gerais (FAPEMIG), Bio-Manguinhos/FIOCRUZ; PROEP/CPqRR/FIOCRUZ; Conselho Nacional de Desenvolvimento Científico e Tecnológico (CNPq), Programa Nacional de Imunizações (PNI), Secretaria de Vigilância em Saúde (SVS) - Ministério da Saúde, Brazil for financial support (Grant\# TC 277/2013). The authors acknowledge valuable expertise help by Luiz Antônio Bastos Camacho, Cleandro Pires de Albuquerque, Luciana Feitosa Muniz and Talita Yokoy de Souza. JGCdR and KT received financial support from CNPq. ACVO received financial support by winning the 2013 PANLAR Rheumatology Award. OAMF and ATC thank the CNPq for the fellowships (PQ).

\section{Funding}

Secretaria de Vigilância em Saúde (SVS) - Ministério da Saúde, Brazil (Grant\# TC 277/2013). JC received financial support from Fundaçāo de Amparo à Pesquisa de MG (FAPEMIG) (Grant\# BPD-00205-13). KT received financial support from Conselho Nacional de Pesquisa (CNPq) (Grant\# 167663/2013-5). AO received financial support by winning the 2013 PANLAR Rheumatology Award.

\section{Availability of data and materials}

The datasets generated and analysed during the current study are available from the corresponding author on reasonable request.

\section{Authors' contributions}

$A O, C D, O M$, and $L M$ conceived of the presented idea. CF, ACC, and VP collected the samples. CF, ACC, JC, LA, KT, LF, IR, and ATC performed the T cell analyses. VP, LF, IR, and ATC performed the B cell analyses. MM and SL performed the PRNT tests. CF, AO, ATC, ACC, LF, IR, OM, and LM analyzed the data and helped with the statistical analyses. CF, ACC, LF, IR, ATC, OM, and LM wrote the manuscript with input from all authors. ATC, OM, and LM supervised the project. All authors discussed the results and contributed to the final version of the manuscript. All authors read and approved the final manuscript.

\section{Ethics approval and consent to participate}

This study was approved by the Ethics Committee for studies with human subjects at Instituto René Rachou FIOCRUZ (CPqRR \# 180911). All subjects gave written informed consent in accordance with the Declaration of Helsinki.

\section{Consent for publication}

Not applicable.

\section{Competing interests}

Two authors (MM and SL) are employees at the 17DD-YF vaccine manufacturer (Bio-Manguinhos, Fundação Oswaldo Cruz), and five authors work in other units of Fundação Oswaldo Cruz (ACC, VP, LA, ATC, and OM). Bias from competing interest was prevented by (1) collaboration of three clinical physicians (CF, AO, and LM) with experience in rheumatic diseases from Universidade de Brasília, (2) one official member from the Brazilian Health Ministry (CD) with experience in vaccine epidemiological vigilance and policies, (3) two independent professionals working as undergraduate students (LF and $I R)$, and (4) two post-doc researchers ( $J C$ and KT) working in the field of infectious diseases. The FIOCRUZ extramural coworkers contributed with the critical overview of the study design, volunteers' immunization and medical care, blood sample collection, blind sample handling and processing, data collection, statistical analysis, and data interpretation.

The authors declare that they have no competing interests.

\section{Publisher's Note}

Springer Nature remains neutral with regard to jurisdictional claims in published maps and institutional affiliations.

\section{Author details}

'Departamento de Reumatologia, Hospital Universitário de Brasília, Universidade de Brasília, Braślia, DF, Brazil. Instituto René Rachou, Fundação Oswaldo Cruz - FIOCRUZ-Minas, Belo Horizonte, MG, Brazil. ${ }^{3}$ Instituto de Tecnologia em Imunobiológicos Bio-Manguinhos - FIOCRUZ, Rio de Janeiro,
RJ, Brazil. ${ }^{4}$ Programa Nacional de Imunizações - Secretaria de Vigilância em Saúde, Ministério da Saúde, Brasília, DF, Brazil. ${ }^{5}$ Grupo Integrado de Pesquisas em Biomarcadores, Instituto René Rachou, Fundação Oswaldo Cruz, FlOCRUZ-Minas, Avenida Augusto de Lima, 1715 Barro Preto, Belo Horizonte 30190-002, Brazil.

Received: 2 September 2018 Accepted: 25 February 2019

Published online: 14 March 2019

\section{References}

1. Araki Y, Mimura T. The mechanisms underlying chronic inflammation in rheumatoid arthritis from the perspective of the epigenetic landscape. J Immunol Res. 2016:6290682. https://doi.org/10.1155/2016/6290682.

2. Spindler A, Bellomio V, Berman A, Lucero E, Baigorria M, Paz S, et al. Prevalence of rheumatoid arthritis in Tucuman. Argentina J Rheumatol. 2002;29:1166-70.

3. Pelaez-Ballestas I, Sanin LH, Moreno-Montoya J, Alvarez-Nemegyei J, BurgosVargas R, Garza-Elizondo M, et al. Epidemiology of the rheumatic diseases in Mexico. A study of 5 regions based on the COPCORD methodology. J Rheumatol Suppl. 2011;86:3-8.

4. Marques-Neto JF, Gonçalves ET, Langen LFOB, Cunha MFL, Radominski S, Oliveira SM, et al. Multicentric study of the prevalence of adult rheumatoid arthritis in Brazilian population samples. Rev Bras Reumatol. 1993;33:169-73.

5. Senna ER, De Barros AL, Silva EO, Costa IF, Pereira LV, Ciconelli RM, et al. Prevalence of rheumatic diseases in Brazil: a study using the COPCORD approach. J Rheumatol. 2004;31:594-7.

6. Meroni PL, Zavaglia D, Girmenia C. Vaccinations in adults with rheumatoid arthritis in an era of new disease-modifying anti-rheumatic drugs. Clin Exp Rheumatol. 2017;36:317-28.

7. Muniz LF, Silva CR, Costa TF, da Mota LM. Vaccination in patients from Brasilia cohort with early rheumatoid arthritis. Rev Bras Reumatol. 2014;54: 349-55.

8. Friedman MA, Winthrop KL. Vaccines and disease-modifying antirheumatic drugs: practical implications for the rheumatologist. Rheum Dis Clin N Am. 2017:43:1-13.

9. Goldani LZ. Yellow fever outbreak in Brazil, 2017. Braz J Infect Dis. 2017;21: 123-4.

10. Ortiz-Martinez Y, Patino-Barbosa AM, Rodriquez-Morales AJ. Yellow fever in the Americas: the growing concern about new epidemics. F1000Res. 2017;6: 398.

11. Simoes M, Camacho LA, Yamamura AM, Miranda EH, Cajaraville AC, SilvaFreire M. Evaluation of accuracy and reliability of the plaque reduction neutralization test (micro-PRNT) in detection of yellow fever virus antibodies. Biologicals. 2012;40:399-404.

12. Miyaji KT, Avelino-Silva VI, Simoes M, Freire MD, Medeiros CR, Braga PE, et al. Prevalence and titers of yellow fever virus neutralizing antibodies in previously vaccinated adults. Rev Inst Med Trop S Paulo. 2017;59:e2 https:// doi.org/10.1590/s1678-9946201759002.

13. Campi-Azevedo AC, Costa-Pereira C, Antonelli LR, Fonseca CT, TeixeiraCarvalho A, Villela-Rezende G, et al. Booster dose after 10 years is recommended following 17DD-YF primary vaccination. Hum Vaccin Immunother. 2016;12:491-502.

14. Luiza-Silva M, Campi-Azevedo AC, Batista MA, Martins MA, Avelar RS, Silveira- Lemos D, et al. Cytokine signatures of innate and adaptive immunity in 17DD yellow fever vaccinated children and its association with the level of neutralizing antibody. J Infect Dis. 2011;204:873-83.

15. Costa-Pereira C, Campi-Azevedo AC, Coelho-dos-Reis JG, PeruhypeMagalhāes V, Araújo MSS, Antonelli LRV, et al. Multi-parameter approach to evaluate the timing of memory status after primary 17DD-YF vaccination. PLoS Negl Trop Dis. 2018. https://doi.org/10.1371/journal.pntd.0006462.

16. Watson AM, Klimstra WB. T cell-mediated immunity towards yellow fever virus and useful animal models. Viruses. 2017;9. https://doi.org/10.3390/ v9040077.

17. Mason RA, Tauraso NM, Spertzel RO, Ginn RK. Yellow fever vaccine: direct challenge of monkeys given graded doses of 17D vaccine. Appl Microbiol. 1973;25:539-44.

18. Julander JG, Trent DW, Monath TP. Immune correlates of protection against yellow fever determined by passive immunization and challenge in the hamster model. Vaccine. 2011;29:6008-16. 
19. Camacho LA, Freire MS, Leal ML, Aguiar SG, Nascimento JP, Iguchi T, et al. Immunogenicity of WHO-17D and brazilian 17DD yellow fever vaccines: a randomized trial. Rev Saude Publica. 2004;38:671-8.

20. Niedrig M, Lademann M, Emmerich P, Lafrenz M. Assessment of lgG antibodies against yellow fever virus after vaccination with 17D by different assays: neutralization test, haemagglutination inhibition test, immunofluorescence assay and ELISA. Tropical Med Int Health. 1999;4: 867-71.

21. Collaborative Group for Studies on Yellow Fever Vaccines. Duration of postvaccination immunity against yellow fever in adults. Vaccine. 2014;32:4977-84.

22. Miller JD, van der Most RG, Akondy RS, Glidewell JT, Albott S, Masopust D, et al. Human effector and memory CD8+ T cell responses to smallpox and yellow fever vaccines. Immunity. 2008;28:710-22.

23. Akondy RS, Monson ND, Miller JD, Edupuganti S, Teuwen D, Wu H, et al. The yellow fever virus vaccine induces a broad and polyfunctional human memory CD8+ T cell response. J Immunol. 2009;183:7919-30.

24. Blom K, Braun M, Ivarsson MA, Gonzalez VD, Falconer K, Moll M, et al. Temporal dynamics of the primary human T cell response to yellow fever virus $17 \mathrm{D}$ as it matures from an effector- to a memory-type response. J Immunol. 2013;190:2150-8.

25. Martins MA, Silva ML, Marciano AP, Peruhype-Magalhaes V, Eloi-Santos SM, Ribeiro JG, et al. Activation/modulation of adaptive immunity emerges simultaneously after 17DD yellow fever first-time vaccination: is this the key to prevent severe adverse reactions following immunization? Clin Exp Immunol. 2007;148:90-100.

26. Neves PC, Matos DC, Marcovistz R, Galler R. TLR expression and NK cell activation after human yellow fever vaccination. Vaccine. 2009;27:5543-9.

27. Silva ML, Espirito-Santo LR, Martins MA, Silveira-Lemos D, PeruhypeMagalhaes V, Caminha RC, et al. Characterization of main cytokine sources from the innate and adaptive immune responses following primary 17DD yellow fever vaccination in adults. Vaccine. 2011;29:583-92.

28. Silva ML, Espirito-Santo LR, Martins MA, Silveira-Lemos D, PeruhypeMagalhaes V, Caminha RC, et al. Clinical and immunological insights on severe, adverse neurotropic and viscerotropic disease following 17D yellow fever vaccination. Clin Vaccine Immunol. 2010;17:118-26.

29. Rakoczi E, Szekanecz Z. Pneumococcal vaccination in autoimmune rheumatic diseases. RMD Open. 2017;3:e000484. https://doi.org/10.1136/ rmdopen-2017-000484. Review.

30. Rubin LG, Levin MJ, Ljungman P, Davies EG, Avery R, Tomblyn M, et al. 2013 IDSA clinical practice guideline for vaccination of the immunocompromised host. Clin Infect Dis. 2014;58:309-18.

31. Gabay C, Bel M, Combescure C, Ribi C, Meier S, Posfay-Barbe K, et al. Impact of synthetic and biologic disease-modifying antirheumatic drugs on antibody responses to the AS03-adjuvanted pandemic influenza vaccine: a prospective, open-label, parallel-cohort, single-center study. Arthritis Rheum. 2011;6:1486-96

32. McMahan $\mathrm{ZH}$, Bingham $\mathrm{CO}$ 3rd. Effects of biological and non-biological immunomodulatory therapies on the immunogenicity of vaccines in patients with rheumatic diseases. Arthritis Res Ther. 2014;16:506.

33. Sociedade Brasileira de Reumatologia. Nota técnica SBR/SBIm/SBI/SBMT: vacina febre amarela em pacientes reumáticos - 31/01/2018. [Portuguese]. 2018. Available from: https://sbim.org.br/images/files/nt-fa-reumato-sbrsbim-sbi-sbmt-180131.pdf. Accessed 2 July 2018.

\section{Ready to submit your research? Choose BMC and benefit from:}

- fast, convenient online submission

- thorough peer review by experienced researchers in your field

- rapid publication on acceptance

- support for research data, including large and complex data types

- gold Open Access which fosters wider collaboration and increased citations

- maximum visibility for your research: over $100 \mathrm{M}$ website views per year

At BMC, research is always in progress.

Learn more biomedcentral.com/submissions 